\title{
Recursive Application of Structural Templates to Efficiently Compress Parsed XML
}

\author{
Akira Kinno $^{1}$, Hideki Yukitomo ${ }^{2}$, Takehiro Nakayama ${ }^{1}$, and Atsushi Takeshita ${ }^{1}$ \\ 1 Multimedia Labs. NTT DoCoMo, Inc., \\ 3-5 Hikari-no-oka, Yokosuka, Kanagawa, Japan \\ \{kinno, nakayama, takeshita\}@mml.yrp.nttdocomo.co.jp \\ 2 Panasonic Mobile Communications Co., Ltd. \\ 600 Saedo-cho, Tsuzuki-ku, Yokohama, Japan \\ Yukitomo.Hideki@jp.panasonic.com
}

\begin{abstract}
This paper proposes an efficient compression method for parsed XML. Our method involves structure-based compression (SBC). In contrast to SBC, our method recursively inputs compressed parsed XML by parsing template instances as a node. Simulation results show that our method can reduce the memory requirements for real XML contents by almost $18 \%$, and retains its compression efficiency even if the application domain changes, while that of SBC varies depending on the structural templates provided. One application of our method is to accelerate the response to native XML database queries.
\end{abstract}

\section{Introduction}

XML is increasingly used as a data format for adapted contents delivery which involves the integration of material from several source XML documents according to the user's preference and device capability.

For storing XML documents, which are targeted for adaptation, native XML databases(NXDBs) are suitable. Because they efficiently support XML-specific capabilities not included in most relational databases(RDBs) currently available. However, NXDB still has considerable points compared to RDBs. One point is at the access level. Large volumes of XML data stored in textural form are hard to access, because parsing is an expensive process. To improve the speed of accessing XML data, caching parsed XML in-memorycan be a promising approach. However, parsed XML has huge memory requirements [4].

In this paper, we propose an efficient method for compressing parsed XML. It uses structure-based compression (SBC) in which the structural information of parsed XML is replaced by structural templates given in advance [5]. Further, it replaces the structures in compressed XML, which also include template instances, with structural templates. This enables higher compression efficiency through the use of small templates, which can be more easily created, than is offered by SBC with the same structural templates. Simulations verify the efficiency of our method, and prove that it well handles even actual XML in terms of the compression ratio.

Eqauations and terms in this paper are refered to Appendix. 


\section{Structure-Based Compression}

With structure-based compression(SBC), parsed XML can be compressed while still supporting the ability of prompt traverse. The basic idea of SBC is to substitute the pure structure with structural templates given in advance [5].SBC is defined as below.

Method 1 Structure-based compression replaces subgraph $G^{\prime} \subseteq G$ with template instance $T^{\prime} \subseteq T_{i}$, when $G^{\prime}=g \subseteq G^{*}$ and joint type of edge, which joins $\forall v \in G^{\prime}$ and $w \in\left(G \cap \overline{G^{\prime}}\right)$, equals $J^{*}$ of isomorphism $\phi(v)=\nu^{*} \in g$.

It is obvious that compression efficiency increases with the number of nodes in the templates and the frequency with which the templates are used. The level of efficiency is, however, somewhat suspect since it strongly depends on the XML data and chosen templates. To make SBC generally practical, we have to identify the set with the best (or quasi-best)structural templates that minimize the total data size of the templates set and compressed XML . In other words, we have to find large and frequent sub-trees in the forest, and this is, in general, a time consuming process [6]. Moreover, SBC has to minimize the data size of the template set. Because SBC must have a template set to extract compressed $\mathrm{XML}$, the best structural templates must offer the minimum data size of template set.

\section{Recursive SBC}

\subsection{The Basic Idea}

Our method avoids the difficulties associated with identifying the best templates; it achieves higher compression efficiency with the use of small template sets, i.e. few structural templates, that have few nodes, than is true with SBC. In general, small template sets are easier to create and their data size can be made reduced. However, in SBC, their compression efficiency is small. Our method is defined below.

Method 2 In Method 1, our method contracts graph $G^{\prime}$ into node $v_{G^{\prime}} \notin G$. That is, our method generates contracted graph $G / G^{\prime}$. Moreover, our method recursively inputs $G / G^{\prime}$ as $G$.

A unique characteristic of our method is that it replaces subgraphs, which also include template instances, with structural templates. In other words, our method replaces subgraphs with combined structural templates. That is, while the data size of template sets is small, the compression efficiency of our method with the small template sets is the same with that of SBC with the large template sets, which consist of templates combined with each template in the small template sets.

Since our recursive matching approach contributes that the number of times that structural templates are applied exceeds that with SBC. Since templates are replaced more frequently, our method has higher compression efficiency than SBC assuming the use of the same template set. 


\subsection{Comparison of Memory Consumption}

To evaluate the efficiency of our method, we compared the memory consumption of parsed XML treated by our method to those required by non-compressed parsed XML and SBC-treated XML. Memory consumption follows the definition by [5]. The memory consumption of non-compressed parsed XML U(x) and that of parsed XML treated by SBC C(x) are given by equations (1)(2)(3).

We acknowledge that our method incurs some recursion overhead because it generates a new node to contract subgraphs for each template application. Thus, our method may be, overall, worse than SBC if the overhead exceeds the gains made in compression efficiency. We define the memory consumption of parsed XML treated by our method $C^{*}(x)$ that is given by equations (4)(5).

\section{Simulation}

\subsection{Conditions}

We conducted a simulation to examine the space efficiency of our method. In this section we compare memory consumption $C(x), C^{*}(x)$ for each site. We implemented our method and SBC using Apache Xalan's DTM. We used a simple algorithm to find every place at which the structural template matched the XML documents. For each XML document, every structural template was tested as to whether it matched the subtree rooted by each node in the depth first manner from leaf node side. After that, our method recursively processed the compressed XML. To solve recursion overhead problem of our method, we applied a simple recursion terminating algorithm; our method compares the current results, i.e., compression ratio, of recursion with the previous results, and if the current results are worse than the previous results, recurison is terminated and the previous results are output.

Table 1. Sample data information

\begin{tabular}{|c|c|c|c|l|}
\hline Site & $\begin{array}{c}\text { \# of } \\
\text { documents }\end{array}$ & $\begin{array}{c}\text { Ave. file } \\
\text { size [KB] }\end{array}$ & $\begin{array}{c}\text { Ave. \# of } \\
\text { nodes }\end{array}$ & contents \\
\hline \hline A & 933 & 27.4 & 1143.7 & rumors, tips about misc. topics \\
\hline B & 629 & 17.0 & 698.2 & technology-related news article \\
\hline
\end{tabular}

As the input XML documents, we collected HTML documents from two web sites (Table 1). By complementing the illegal notation in HTML documents, we translated them to valid XML documents. Some elements or tags of the documents were complemented in advance to yield validated XML. Note that, we treated a single HTML file as a single document in this simulation. We carefully chose 6 structural templates to compare our method with SBC (Figure 1). The rule of making our templates is that when we combine our templates by using recursive SBC, we get templates used in [5]. 


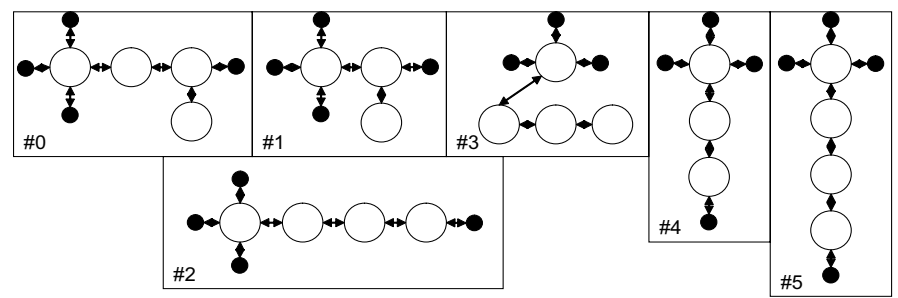

Fig. 1. Structural templates used in simulation. Filled and hollow circles represent open-joint information and nodes, respectively. The relationships are represented by arrows. Note that, we took care of components of templates' open-joint information for Apache Xalan to permit parsing of the compressed XML

\subsection{Simulation Results and Discussion}

The simulation results show that our method is better than SBC(Figure 2). In terms of average compression ratio, moreover, its compression efficiency remains high even if the application domain changes, while that of SBC falls depending which structural templates were given in advance. Considering the use of our method in NXDB, the latter result supports our contention that our method is better than SBC. In general, the structures of XML data in NXDB are defined by various schema to better utilize the NXDB characteristic, which is that NXDB can treat native XML data. In this case, our method performs more efficiently than SBC. Moreover we discuss the relationship between NXDB performance and compression ratio. Assuming that the time taken to put XML data into memory is much shorter than that taken for XML processing, the average processing speed without any compression method $S$ is given by equation (6). On the other hand, the average processing speed with compression method $S^{\prime}$ is given by equation (7). Thus, the progress rate of processing speed by compression $R$ is given by equation (8). If cache hit ratio $h$ and parsing cost $r$ are high, equation (8) shows that $R$ can be made very high by increasing compression rate $C_{r}$. This assumption is reasonable when querying NXDB for two reasons. The first reason is that parsing is an expensive process and unsuitable when ran-
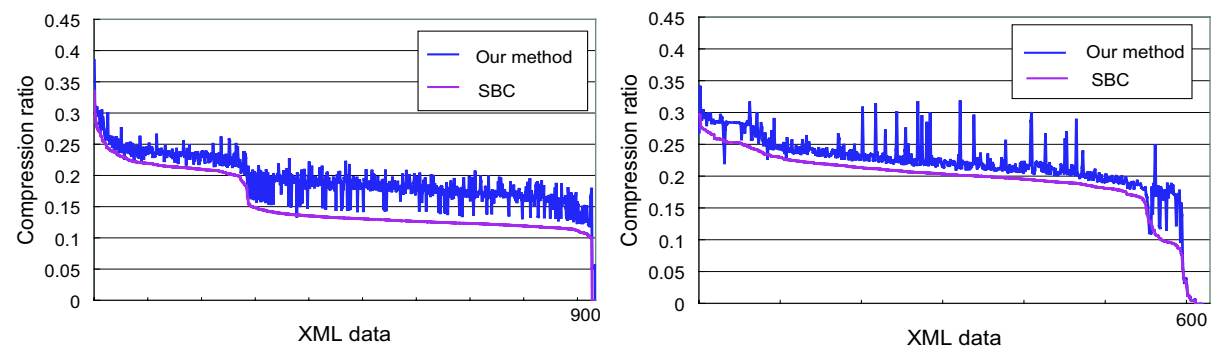

Fig. 2. Compression ratios of compressed pared XML created by our method and SBC for the documents of sites A (a) and B (b).The average compression ratios are written in parentheses 
domly accessing individual elements of a data structure (i.e., querying process). The other is that caching XML documents is effective, because we can assume the locality of data querying from NXDB is high.

Other approachs to improve the speed of XML process, [3], [2] and [1] are also text based approaches, but aim at query-enabling XML compression. However, they do not well support content adaptation because content adaptation requires the partial addition, deletion, and alternation of XML documents. To support these features, the whole XML document should be kept in parsed form, and the compression of parsed XML should be considered.

\section{Conclusion}

This paper proposed an efficient method for compressing parsed XML. It replaces the structures in compressed XML, which also includes template instances, with structural templates. This enables higher compression efficiency through the use of small templates than is possible with structure-based compression (SBC) [5] with the same structural templates. Simulations showed that our compression ratios were better than those of $\mathrm{SBC}$ in the two actual sites examined. Moreover, they showed that our method keeps its compression efficiency even if the application domain changes, unlike SBC since the latter must use the structural templates given in advance.

Our future work includes evaluating the impact of our method on the time needed for querying. This paper used only simulations to examine the compression efficiency and why the compression of parsed XML can accelerate query speeds. We will elucidate how much our method can decrease the time needed for querying.

\section{References}

1. J. Cheng and W. Ng, "XQzip: Querying Compress XML Using Structural Indexing".In proc. of Extending Database Technology, 2004.

2. J.K. Min, M.J. Park, and C.W. Chung, "XPRESS: A Queriable Comression for XML Data". In proc. of ACM Symp. on the Management of Data, 2003.

3. P.M. Tolani and J.R. Haritsa,"XGRIND:A Query-friendly XML Compressor". In proc.of Int. Conf. of Data Engineering, 2002.

4. XSLT Processor Xalan, http://xml.apache.org/xalan-j/index.html.

5. H. Yukitomo, A. Kinno, T. Nakayama and A. Takeshita, "Structure-based compression of parsed XML for Content Adaptation". In proc. of the 49th IPSJ SigDD workshop, Tokyo, March 2005.

6. M. J. Zaki, "Efficiently mining frequent trees in a forest". In proc. of the 8th ACM SIGKDD, Edmonton, 2002.

\section{Appendix: Terms and Equations}

Terms in this paper:

Parsed XML is a graph $G_{s}=\left(V_{s}, E_{s}\right)$. Each node $v_{s} \in V_{s}$ has node type, name, and value. Each edge $e_{s} \in E_{s}$ has relationships. 
Relationship is a set of edge types between nodes, i.e., parent, first child, and previous and next sibling.

Pure structure is a graph $G=(V, E)$, where $E=(\epsilon, J)$, where $\epsilon \subseteq[V]^{2}$, and $J$ is a set of relationships. In contrast to parsed $X M L$, it is unconcerned with node type, name and value.

Open-joint information is a set of references between a node included in a structural template and an outside node. Let open-joint information $E x=\left(\nu^{*}\right.$, $\left.J^{*}\right)$, where $\nu^{*} \subseteq V^{*}$ and $J^{*}$ is the relationship between $\forall v \in \nu^{*}$ and $w \in\left(V \cap \overline{V^{*}}\right)$. Structural template is a data structure to represent a fragment of the pure structure. Let a structural template $\mathrm{T}=\left(T_{i d}^{*}, G^{*}, E x\right)$, where $T_{i d}^{*}$ is a set of template identifiers, and $G^{*}$ is a set of fragments of the pure structure $G$.

Template instance is a data structure to represent a part of parsed XML in compressed XML. It consists of an instance identifier $T_{i d}$, a reference to the structural template $T_{i d}^{*}$, node information $V_{t}$, and outer joint information $E_{t}$. Let a template instance $T_{i}=\left(T_{i d}, T_{i d}^{*}, V_{t}, E_{t}\right)$.

Equations $^{1}$ in this paper:

$$
\begin{aligned}
U(x)= & 4 \cdot 4 N_{x}+4 \cdot 2 N_{x}+D(x), \\
C(x)= & 4\left(\sum A\left(t_{i}, x\right)\left(J\left(t_{i}\right)+2\right)+4 M_{x}\right)+4 \cdot 2 N_{x}+D(x), \\
M_{x}= & N_{x}-\sum A\left(t_{i}, x\right) \cdot N_{t_{i}}, \\
C^{*}(x)= & 4\left(\sum A^{*}\left(t_{i}, x\right)\left(J\left(t_{i}\right)+2\right)+4 M_{x}^{*}\right)+4 \cdot 2 N_{x}+D(x), \\
M_{x}^{*}= & N_{x}-\sum A^{*}\left(t_{i}, x\right) \cdot N_{t_{i}}+\sum A^{*}\left(t_{i}, x\right), \\
S= & ((1-r) W) h+(r \cdot W+(1-r) W)(1-h), \\
S^{\prime}= & (r \cdot W+(1-r) W)\left(1+C_{r}\right) h \\
& +(r \cdot W+(1-r) W)\left(1-\left(1+C_{r}\right) h\right), \\
R= & \frac{S-S^{\prime}}{S}=\frac{h \cdot r}{1-r \cdot h} \cdot C_{r}
\end{aligned}
$$

1 Explanatory note: $U(x)$ :memory consumption of non-compressed content, $C(x)$ :that of content treated by SBC, $C^{*}(x)$ :that of content treated by our method, $N_{x}$ :\# of nodes in content $x, D(x)$ :the sum of string data, $M_{x}$ : \# of nodes for which no template was applied, $t_{i}: i$-th structural template, $A(t, x)$ :\# of times structural template $t$ occurs in content $x, J(t)$ is the size of joint information for structural template $t$, $M_{x}^{*}$ :\# of nodes to which no template was applied even our method applying, $A^{*}: \#$ of times structural template occurs in content, $S$ :average processing speed without any compression method, $S^{\prime}$ :that with compression method, $R$ :progress rate of that by compression, $W$ :the whole XML processing speed, $r$ :the time cost ratio of the parsing process among all processes, $h$ :the cache hit ratio, and $C_{r}$ :the compression ratio 